\title{
THE ANALYSIS OF ROCK SURFACE ASPERITIES
}

\author{
Dalibor Martisek, Jana Prochazkova \\ Brno University of Technology, Faculty of Mechanical Engineering \\ Institute of Materials Science and Engineering, NETME centre \\ Technicka 2, Brno \\ Czech Republic \\ martisek@fme.vutbr.cz,prochazkova.j@fme.vutbr.cz
}

\begin{abstract}
The analysis of rock surface is commonly used in technical practice. The nature discontinuity rocks are often represented by specific degree of roughness. This value has the significant effect on the rock slope stability. It is defined by the asperities and the computation is based on the visual comparison of discontinuity traces or discontinuity surfaces with standard two-dimensional graphs. The visual comparison is a subjective method, so that we propose novel method for roughness-angles analysis. Our method consists of the application of cosine Fourier series. We tested the method on the sample of limestone. The article also describes the method Surface from Focus that reconstruct the 3D surface from a set of partially focused images.
\end{abstract}

Keywords: surface roughness, rock surface analysis, datum plane, wave analysis, tilt angle, Linderberg-Levi theorem.

\section{Introduction}

Study of surface asperities is important in many branches. Papers [1, 2] deal with roughness of machined metal surfaces for example. The rock joint surface roughness is an important factor that influences the shear strength of rock masses considerably so that it is critical for the mechanical stability of different civil engineering constructions. The shear strength of a discontinuity in a soil or rock mass may have a strong impact on its mechanical behaviour ([3-7] for example). The pressure of gasses and fluids along the discontinuity, infill materials or the shape of discontinuity surfaces determine this shear strength [8-11]. The sliding criterion that is the part of Slope Stability Classification [12] is often used for the properties estimation of the shear strength of discontinuity. The criterion is based on visual and tactile characterization $[13,14]$.

The physical definition says that the slope is given by a theoretical inclined datum plane which is used to minimize or eliminate local topographic and near-surface effects of a primary plane [15]. The tilt-angle refers to the angle given by the inclined datum plane and horizontal plane or precisely, by the angle of the datum plane normal and the vertical direction (Fig. 1, left). In geotechnical practice, the tilt-angle is measured by a tilt-test [16, 17]. Two pieces of rock containing a discontinuity are held horizontal and the sample is slowly tilted until the top block moves (Fig. 1, left).

Let $i$ be the roughness angle (see Fig. 1, right), the detailed description is e.g. in [18, 19]. We differentiate two orders of roughness angles: roughness-angles for first-order asperities that are measured on decimetre scale and roughnessangles for second-order asperities that are measured on centimetre scale [18]. The explanation of these two types is in Fig. 1. right.

The roughness evaluation is based on visual comparison of the trace (the length approx. $1 \mathrm{~m}$ ) or surface (the area approx. $1 \times 1 \mathrm{~m}^{2}$ with the template graphs (Fig. 2, left). These graphs describe the rock property called waviness.
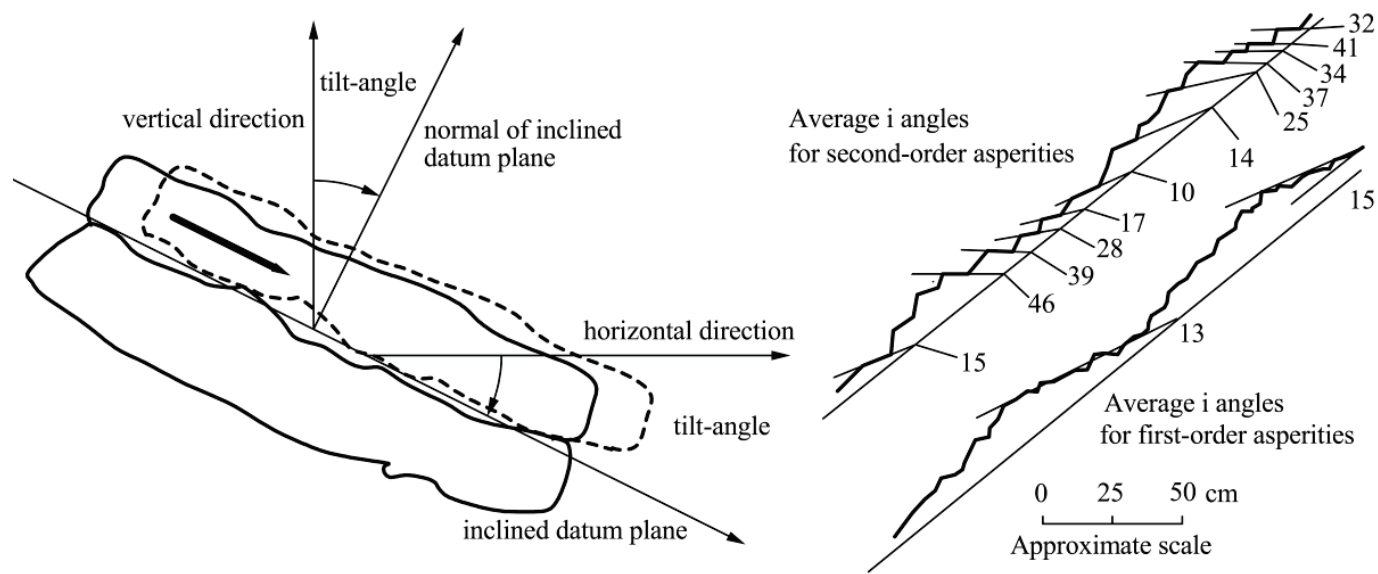

Figure. 1: The illustration of the inclined datum plane and the tilt-angle (left) and roughness angles for first- and second-order asperities (right). 


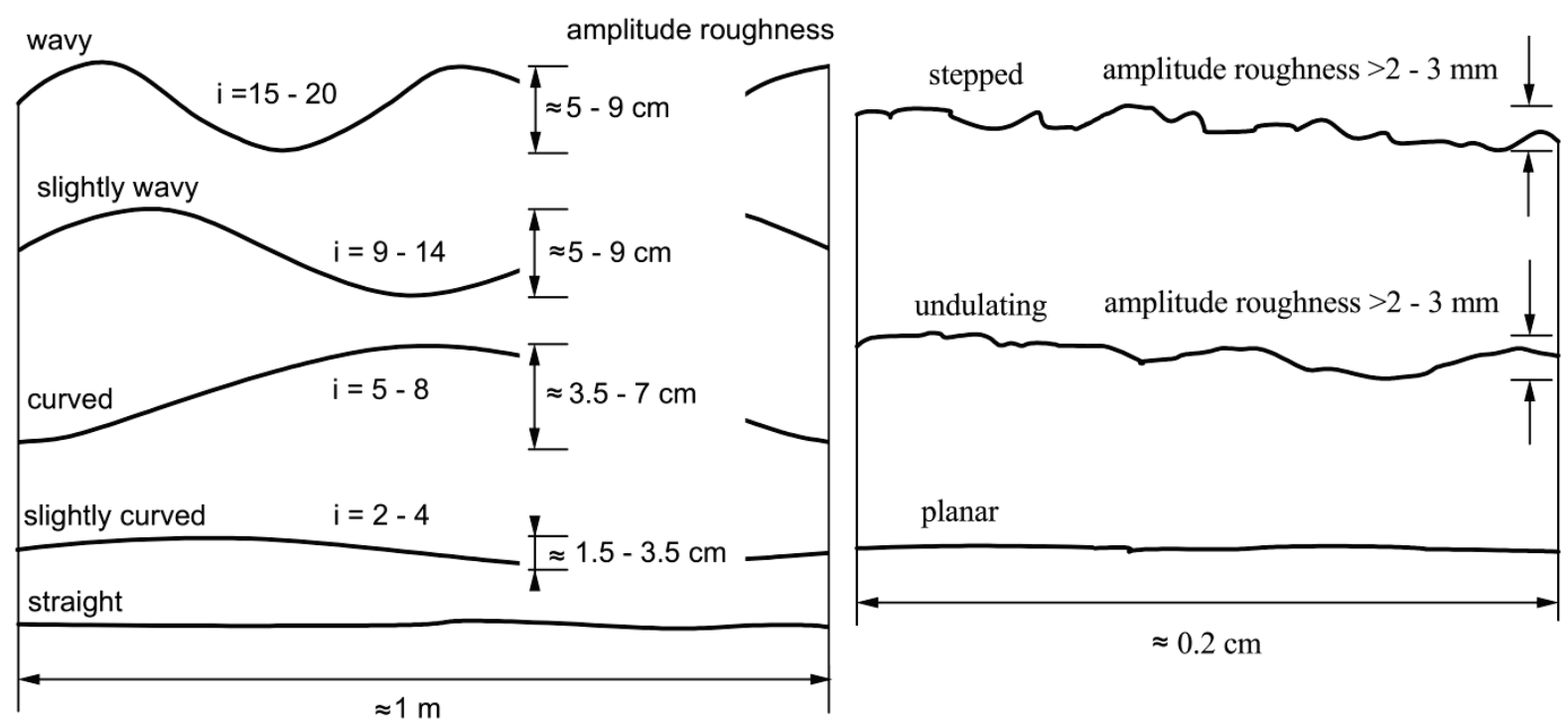

Figure 2: Large scale (left) and small scale (right) templates of the roughness graphs, $i$ is a roughness-angle (angles and dimensions are indicative only).

The small-scale roughness is determined visually as well. The trace (the approx. length $0.2 \mathrm{~m}$ ) or surface (the approx. area $0.2 \times 0.2 \mathrm{~m}^{2}$ ) of a discontinuity is compared to the template graphs in Fig. 2, right [6]. This rock property is called undulates.

Let $P$ be the primary rock surface given by the sum of three surfaces: the datum plane $D$, the waved surface $W$ and the undulated surface $U$.

$$
P(i, j)=D(i, j)+W(i, j)+U(i, j)
$$

where $P(i, j) ; D(i, j) ; W(i, j) ; U(i, j)$ are the heights of the corresponding surface point $(i, j)$. The situation for onedimensional case is illustrated in Fig. 3.

In this article, we present the objective method for the datum plane $D$ assignment, measurement of the tilt-angle and the construction of the waved surface $W$ and the undulated surface $U$.

\section{Reconstruction and Analysis Methods}

This section presents reconstruction method Shape from Focus (sec. 2.1) that is based on the statistical method. This method computes a 3D shape of the arbitrary surface from set of images with different focusing. The following sections 2.2 and 2.3 present the analysis method using wave analysis. This evaluation offers sophisticated method to surface asperities determination.
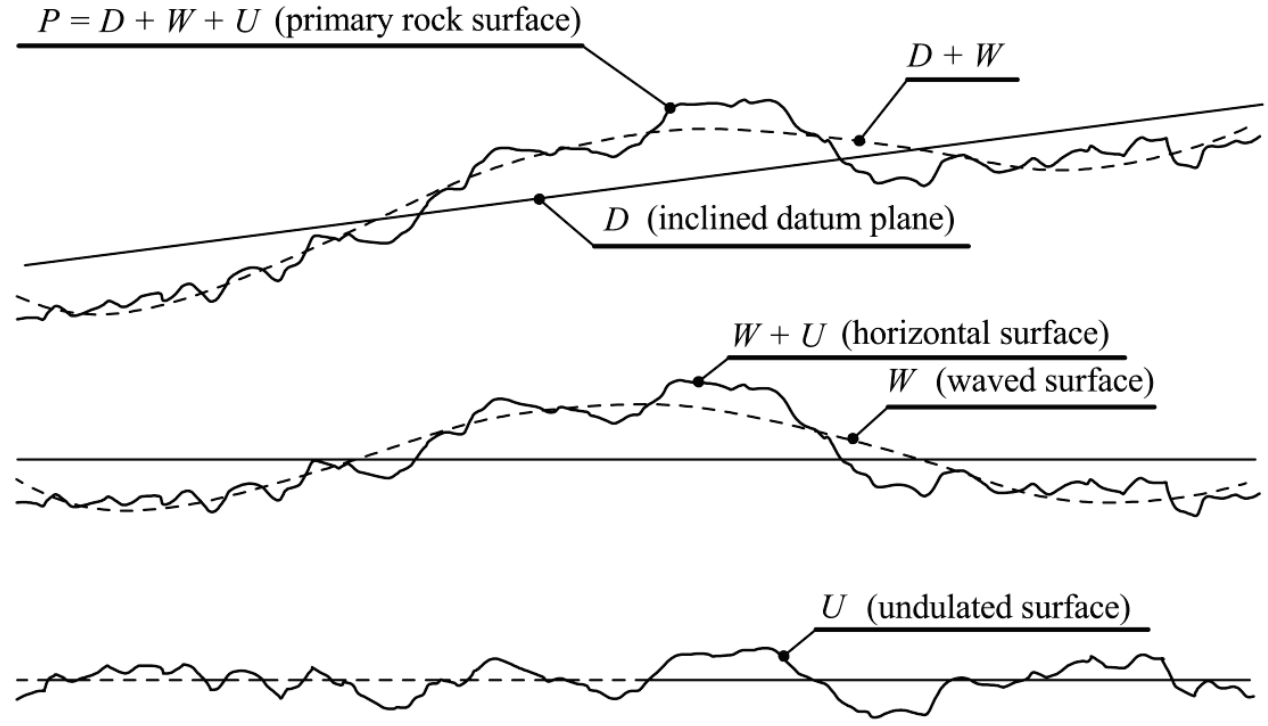

Figure 3: Primary rock surface $P$ is composed of the datum plane $D$, the waved surface $W$ and the undulated surface $U$ (one-dimensional illustration) 


\subsection{Method Shape from Focus}

Proposed method Shape from Focus (SfF) recovers the 3D profile of the arbitrary surface from the set of partially focused images. The process starts with image registration based on cross correlation [20] where we detect the transformations (translation, rotation, scaling) between images.

The phase correlation became a powerful tool for registration of particular focussed images. Let $f_{1} ; f_{2}$ are real functions, then the phase correlation $P_{f_{1} ; f_{2}}(x ; y)$ it is defined as

$$
P_{f_{1} ; f_{2}}(x ; y)=\mathcal{F}^{-1}\left\{\frac{F_{1}(\xi ; \eta) \cdot \bar{F}_{2}(\xi ; \eta)}{\left|F_{1}(\xi ; \eta)\right| \cdot\left|F_{2}(\xi ; \eta)\right|}\right\}
$$

where $F_{1} ; F_{2}$ are the discrete Fourier transforms of $f_{1} ; f_{2}$ (images to be registered), expression $\bar{F}_{2}(\xi ; \eta)$ is the complex conjugate of $F_{2}$. The phase correlation of the real functions $f_{1} ; f_{2}$ is the real function [21]. This property enables us to search for extremes of the phase-correlation function.

Two-dimensional $\delta$-distribution $\delta(x ; y)$ is a limit of a sequence of functions $\delta_{n}(x ; y) ; n \in \mathbb{N}$ such that

$$
\lim _{n \rightarrow \infty} \int_{-\infty}^{\infty} \int_{-\infty}^{\infty} \delta_{n}(x ; y) \mathrm{d} x \mathrm{~d} y=1 ; \quad \lim _{n \rightarrow \infty} \frac{\delta_{n}\left(x_{0} ; y_{0}\right)}{\lim _{(x ; y) \rightarrow(0 ; 0)} \delta_{n}(x ; y)}=0 ; \quad\left(x_{0} ; y_{0}\right) \in \mathbb{R}^{2}-\{(0 ; 0)\}
$$

If two functions are shifted in arguments, i. e. $f_{2}(x ; y)=f_{1}\left(x-x_{0} ; y-y_{0}\right)$, their Fourier transforms are shifted in phase and their phase-correlation function is the $\delta$-distribution shifted in arguments by the opposite shift vector. This is the main idea of phase correlation.

The phase-correlation function can be also used for estimation of image rotation and rescale. If functions $f_{1}$; $f_{2}$ are rotated, shifted and scaled in arguments, i. e.

$$
f_{2}(x ; y)=f_{1}\left(\alpha(x \cos \theta-y \sin \theta)-x_{0} ; \alpha(x \sin \theta+y \cos \theta)-y_{0}\right)
$$

their Fourier spectra and amplitude spectra are related as follows:

$$
\begin{gathered}
F_{2}(\xi ; \eta)=\frac{1}{\alpha^{2}} \exp \left(-i\left(\xi x_{0}+\eta y_{0}\right)\right) \cdot F_{1}\left(\frac{1}{\alpha}(\xi \cos \theta-\eta \sin \theta) ; \frac{1}{\alpha}(\xi \sin \theta+\eta \cos \theta)\right) \\
A_{2}(\xi ; \eta)=\frac{1}{\alpha^{2}} A_{1}\left(\frac{1}{\alpha}(\xi \cos \theta-\eta \sin \theta) ; \frac{1}{\alpha}(\xi \sin \theta+\eta \cos \theta)\right)
\end{gathered}
$$

The shift results in a phase shift, the spectra are rotated in the same way as the original functions and scaled with a reciprocal factor. A crucial step here is amplitude spectra transformation into the logarithmic-polar coordinate system

$$
\exp \rho=\sqrt{x^{2}+y^{2}} ; \quad x=\exp \rho \cos \varphi ; \quad y=\exp \rho \sin \varphi
$$

to obtain $A_{1}^{p} ; A_{2}^{p}: \mathbb{R}_{0}^{+} \times\langle 0 ; 2 \pi) \rightarrow \mathbb{R}_{0}^{+}$such that $A_{2}^{1 p}(\rho ; \varphi)=A_{1}^{1 p}(\rho-\ln \alpha ; \varphi+\theta)$.

Both rotation and scale change were transformed to a shift. The unknown angle $\theta$ and unknown factor $\alpha$ can be estimated by means of the phase correlation applied on the amplitude spectra $A_{1}^{1 p} ; A_{2}^{1 p}$ in the logarithmic-polar coordinate system. After rotating function $f_{2}$ back by the estimated angle $\theta$ and scaling by factor $\alpha$, the shift vector $\left(x_{0} ; y_{0}\right)$ is detected and corrected.

Further, we use cosine Fourier transform for focused areas detection. So, the idea is to perform an even periodic extension of the pixel neighbourhood. This transform does not suffer by edge jump. The expression

$$
{ }^{(k)} F_{i ; j}=\sum_{m=-H}^{H-1} \sum_{n=H}^{H-1}\left[\left|{ }^{(k)} C(i+m ; j+n)\right| \sin ^{2}\left(\pi \frac{\sqrt{m^{2}+n^{2}}}{H}\right)\right]
$$

provides the best results $\left({ }^{(k)} C(i ; j) \mid\right.$ is the value of the amplitude spectrum of the cosine Fourier transform in pixel $[i ; j]$ of the $k$-th image) [22]. Focused 2D image is the composition of the particular pixels with maxima of the focusing criterion.

Afterward, we reconstruct 3D model of the sample. We suppose that all point in the same optical cut have the same height and we compute the stair approximation of the object. We also add a low-pass filter method to refine the results, but low-pass filters do not differentiate whether the high-frequency information is a useful signal or the noise. As the consequence, some details are missing, or unfocused parts appear in the final image [23].

Let $\left\{{ }^{(k)} F_{i ; j}\right\} ; k=1 ; 2 ; . ; ; n$ be a series of random variables, where $F$ evaluate the "height" of pixel $[i ; j]$. For each pixel $[i ; j]$, we can construct theoretically infinitely many probablity distribution functions $p_{i j}^{(r)}$ using different exponents $r$ applied to series members ${ }^{(k)} F_{i ; j}$ : 


$$
p_{i j}^{(r)}(k)=\frac{{ }^{(k)} F_{i ; j}^{r}}{\sum_{s=1}^{n}{ }^{(s)} F_{i ; j}^{r}}
$$

Expected values $P_{i j}^{(r)}$ of random variables $p_{i j}^{(r)}(k)$ given by these probablity functions estimate the height $h_{i j}^{(r)}$ of surface in its pixel $[i ; j]$ :

$$
h_{i j}^{(r)}=E\left(P_{i j}^{(r)}\right)=\sum_{k=1}^{n} k \cdot p_{i j}^{(r)}(k)=\sum_{k=1}^{n} k \cdot \frac{{ }^{(k)} F_{i j}^{r}}{\sum_{s=1}^{n}(s) F_{i ; j}^{r}}
$$

See [21] for more information about SfF method. The other possible reconstruction methods are presented e. g. in [24, $25,28]$.

We tested SfF method on the sample of hydrated cement paste. The tested data were acquired by confocal microscope Olympus OLS 3100. First 3D relief $f_{1}$ was computed by Olympus factory software from confocal scanning data (400 cuts). Second relief $f_{2}$ was constructed by method SfF presented in Sec. 2.1. from non-confocal data (40 images). Due to the porous property of the material, there was the obvious noise in the data, so that this noise was decreased by usage of Lindeberg-Levi Central Limit theorem [25].

We made a comparison of reconstructed surfaces $f_{1}$ and $f_{2}$. The average difference is

$$
A D\left(f_{1} ; f_{2}\right)=8.2432 \mu m
$$

It means that average absolute error is $\pm 4.1216 \mu \mathrm{m}$ per pixel and average relative error is $0.4 \%$ per pixel because the average heigh was $\bar{f}_{1} \approx \bar{f}_{2} \approx 1031 \mu \mathrm{m}$. Our method SfF gives very good results in comparison with implemented software algorithms (note that it works with confocal data).

\subsection{The Computation of the Datum Plane and the Tilt-Angle}

The computation of the datum plane is based on the least squares method. The equation of the plane is given by:

$$
z(x ; y)=a x+b y+c .
$$

We minimize the function through the measured points $\left[x_{i j} ; y_{i j} ; z_{i j}\right]$ :

$$
H(a ; b ; c)=\sum_{i=0}^{W-1} \sum_{j=0}^{H-1}\left(z_{i j}-a x_{i j}-b y_{i j}-c\right)^{2}
$$

where $\left[x_{i j} ; y_{i j} ; z_{i j}\right]$ are points in the image and $W \times H$ is the resolution of the sample. Using the coefficients $a, b, c$, the tilt angle $\alpha$ can be expressed by:

$$
\alpha=\arccos \frac{\mathbf{n} \cdot \mathbf{v}}{\|\mathbf{n}\|}=\arccos \frac{1}{\sqrt{a^{2}+b^{2}+1}}
$$

The negative gradient of the datum plane:

represents the steepest descent of the plane.

$$
-\operatorname{grad} z(x ; y)=-\left(\frac{\partial z}{\partial x} ; \frac{\partial z}{\partial y}\right)=-(a ; b)
$$

\subsection{Wave Analysis}

The evaluation based on the visual comparison of the trace or the surface with the template graphs (Fig. 2) is very subjective and unjustifiable reductive. We propose the method based on cosine Fourier series [25] that can increase the exactness and objectivity of the evaluation. Let

$$
p(x, y)=\sum_{k=0}^{K-1} \sum_{n=0}^{N-1} a_{k n} \cdot \cos \frac{k \pi x}{W} \cdot \cos \frac{n \pi y}{H}
$$

be a Fourier cosine series where

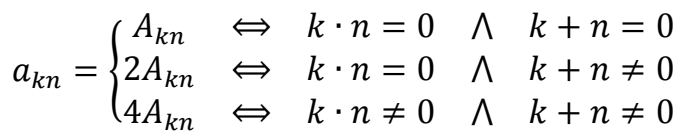

$$
\begin{aligned}
& A_{k n}=\frac{1}{W \cdot H} \iint_{\Omega} f(x ; y) \cos \frac{k \pi x}{W} \cos \frac{n \pi y}{H} \mathrm{~d} x \mathrm{~d} y ; \quad \Omega=\langle 0 ; W\rangle \times\langle 0 ; H\rangle
\end{aligned}
$$




\section{Results and Discussion}

This part shows the application of the proposed 3D reconstruction method SfF and following analysis on real sample of limestone. The sample size is $1 \times 1 \mathrm{~m}$, the photos were made in locality Brno-Hády, Czech Republic. We use the series of the same rock surface images with different focusing for a non-destructive evaluation. Each point of the surface should be focused at least at one image (in the ideal case). The Fig. 4 shows two partly focused images of the limestone surface where the different sharp parts are clearly recognizable. The photos were acquired by photographic camera Canon EOS 600D, the objective EF $100 \mathrm{~mm}$ f/2.8 Macro USM. It was mounted on a tough stand which enables movement in the horizontal direction. The recording device requirement is low depth of focus (DOF).

The images are proceeded by SfF method described in sec. 2.1. The focused parts (in altitude pseudo colours) of ten partly focused image are in Fig. 5 (left). The composed fully focused image with true colours is in Fig. 5 (right).
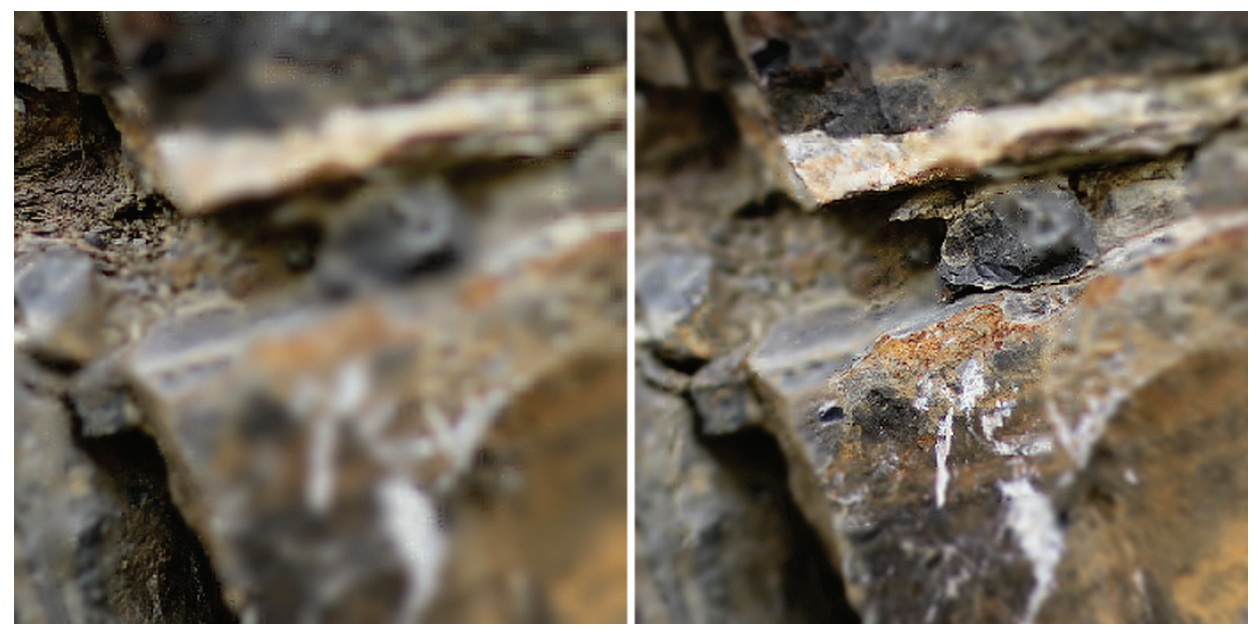

Figure 4: Two partial focused images of the same part of limestone surface. Real size of original data is $1 \times 1 \mathrm{~m}$ (locality Brno-Hády, Czech Republic, photos were acquired by Tomas Ficker).
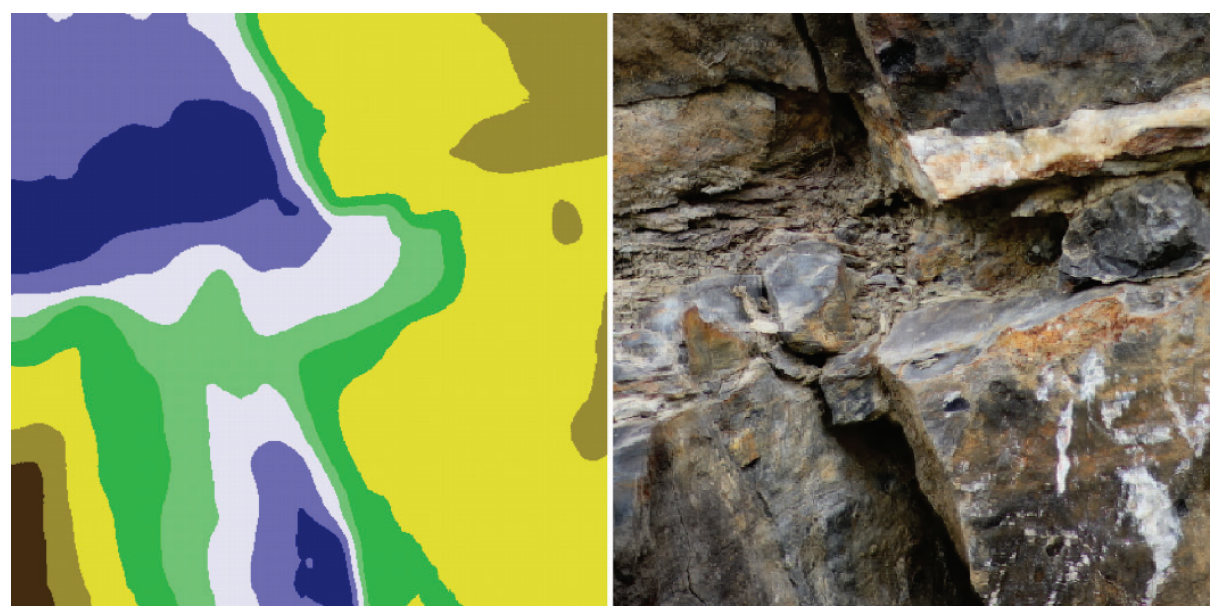

Figure 5: The sharp parts of the series of ten partly focused image (optical cuts) identified by Shape from Focus method using altitude pseudo colours (left) and fully focused image composed from multifocal images with true colours (right). 

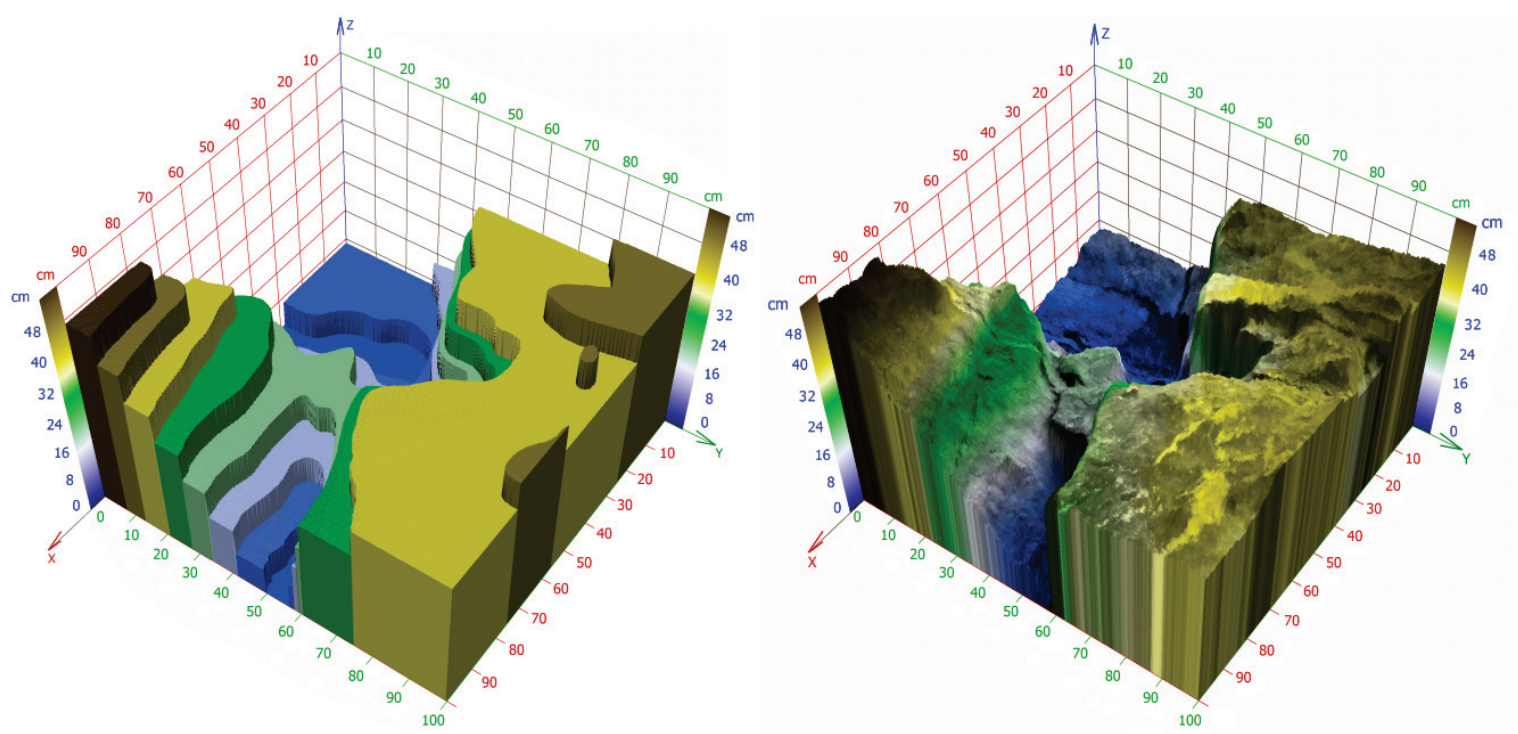

Figure 6: On the left: the stair-approximation of the $3 D$ object: all points belonging to the same optical cut have the same height - the height of the corresponding zone of sharpness. On the right: $3 D$ reconstruction using calculation of expeced values of focusing criteria based on Fourier transform (altitude pseudo colors).
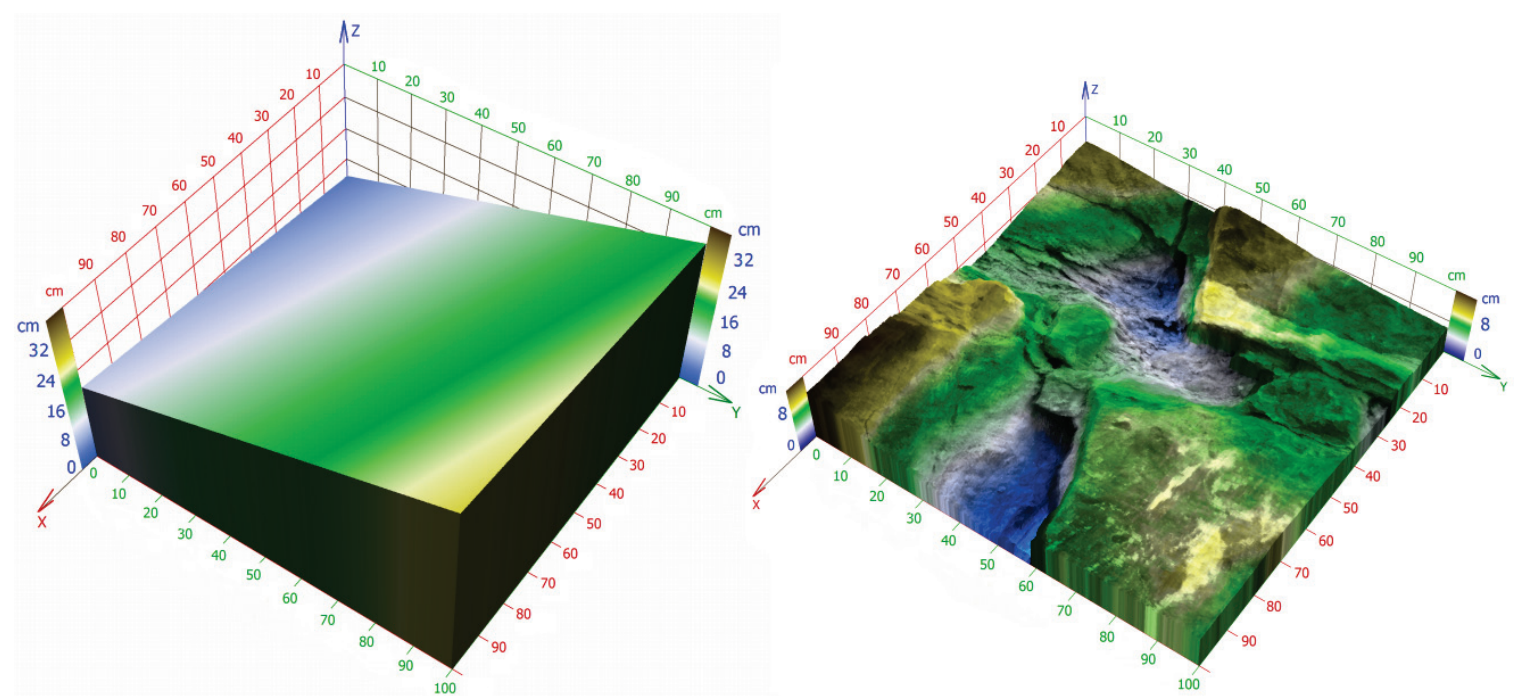

Figure 7. The datum plane (on the left); waved surface (on the right) of the sample from Fig. 6.

Afterward, we reconstruct 3D model of the sample. The corresponding stair approximation of the object is in Fig. 6, (left). The reconstructed surface using the expected values computed by Eq. (9) is in Fig. 6 (right).

Also, we found the equation of the datum plane D (see Fig. 7, left)

$$
D(x ; y)=0.0644 x+0.2282 y+12.8623
$$

The tilt-angle of this plane is $13^{\circ} 21^{\prime}$ and direction of the greatest slope line is $\operatorname{grad} D=(0.0644 ; 0.2282)$. These values were computed using the methods described in Sec. 2.2.

The definition of the surface waviness is the other problem. As is shown in Fig. 1, the large roughness graphs presume that there is one "indicative" and "predominate" wave in the analysed profile. However, this assumption is not fulfilled in real samples data. The shortest wavelength in Fig. 1 (left) is approximately $0.45 \mathrm{~m}$. Therefore, we propose to replace these "indicative" graphs with cosine Fourier series presented in Sec. 2.3. We use the sum of five members of the expression:

$$
p(x, y)=\sum_{k=0}^{K-1} \sum_{n=0}^{N-1} a_{k n} \cdot \cos \frac{k \pi x}{W} \cdot \cos \frac{n \pi y}{H}
$$


where wavelength of the fifth member is $0.4 \mathrm{~m}$. Large scale roughness graphs can be replaced by a waved surface partial sum for $K=N=6$ members. The discrete wavelengths amplitudes of the measured sample are summarized in Table 1 (in centimetres).

Table 1: Wavelengths of individual addends in Eq. (17) and their amplitudes

\begin{tabular}{llllllll}
\hline Sum & & $\mathbf{0}$ & $\mathbf{1}$ & $\mathbf{2}$ & $\mathbf{3}$ & $\mathbf{4}$ & $\mathbf{5}$ \\
\hline & Wavelength & & 200.0 & 100.0 & 66.67 & 50.0 & $\mathbf{4 0 . 0}$ \\
\hline $\mathbf{0}$ & & 5.45450 & 0.03279 & 0.81198 & 0.27976 & 0.42463 & 0.08407 \\
$\mathbf{1}$ & 200.0 & 0.00093 & 0.54961 & 0.37120 & 0.45120 & 0.30554 & 0.00245 \\
$\mathbf{2}$ & 100.0 & 0.53344 & 0.51518 & 0.11513 & 0.26050 & 0.10920 & 0.07675 \\
$\mathbf{3}$ & 66.67 & 0.05779 & 0.13916 & 0.38641 & 0.15127 & 0.17609 & 0.12241 \\
$\mathbf{4}$ & 50.0 & 0.10719 & 0.17163 & 0.25111 & 0.27879 & 0.15115 & 0.10054 \\
$\mathbf{5}$ & 40.0 & 0.10397 & 0.09174 & 0.04907 & 0.02994 & 0.03103 & 0.03403 \\
\hline
\end{tabular}

The roughness-angles for first-order asperities and second-order asperities were measured in the direction of the greatest slope line according to Eq. (14). For this measurement, horizontal surface $W+U$ was used (see Fig. 3). In this surface, peaks with horizontal distance $0.1 \mathrm{~m}$ in the direction of the negative datum plane steepest descent were selected in case of the first-order asperity. The peaks with distance $0.01 \mathrm{~m}$ (defined in the same way) in case of the second-order asperity. In Table 2, distribution of roughness-angles for first-order asperities are summarized. The tabular value corresponds to the sum of column and row degree value.

Table 2: The distribution of roughness-angles for first-order asperities. Bold value 462 means that sample peaks have the roughness-angle equal to $54^{\circ}$.

\begin{tabular}{lcccccccccc}
\hline & $\mathbf{0}$ & $\mathbf{1}$ & $\mathbf{2}$ & $\mathbf{3}$ & $\mathbf{4}$ & $\mathbf{5}$ & $\mathbf{6}$ & $\mathbf{7}$ & $\mathbf{8}$ & $\mathbf{9}$ \\
\hline $\mathbf{0}$ & 512 & 3923 & 7684 & 10906 & 12251 & 12155 & 11589 & 11418 & 10148 & 9113 \\
$\mathbf{1 0}$ & 8224 & 7432 & 6872 & 6494 & 6275 & 6204 & 5913 & 6398 & 6372 & 6128 \\
$\mathbf{2 0}$ & 5888 & 4960 & 4212 & 3944 & 3461 & 3411 & 2996 & 2644 & 2521 & 2421 \\
$\mathbf{3 0}$ & 2100 & 1741 & 1650 & 1612 & 1329 & 1211 & 1188 & 1186 & 1178 & 1014 \\
$\mathbf{4 0}$ & 986 & 878 & 805 & 826 & 927 & 910 & 807 & 706 & 686 & 641 \\
$\mathbf{5 0}$ & 582 & 521 & 531 & 514 & $\mathbf{4 6 2}$ & 449 & 477 & 451 & 442 & 407 \\
$\mathbf{6 0}$ & 367 & 367 & 438 & 495 & 328 & 263 & 202 & 197 & 164 & 155 \\
$\mathbf{7 0}$ & 161 & 138 & 119 & 134 & 125 & 104 & 125 & 91 & 55 & 25 \\
$\mathbf{8 0}$ & 8 & 2 & 2 & 3 & 15 & 15 & 13 & 21 & 371 & 487 \\
\hline
\end{tabular}

\section{Conclusion}

The article presents two approaches to surface evaluation: 3D reconstruction and the roughness analysis. We describe the method Shape from Focus that can reconstruct the arbitrary surface from partially focused images. The method is based on probability distribution functions and its expected value. We tested the method on the small size model of the fracture of cement concrete because it is possible to compare the computed results with correct confocal measurements. We claimed the average absolute error equal to $\mathbf{\pm 4 . 1 2 1 6} \boldsymbol{\mu m}$ per pixel and average relative error $\mathbf{0 . 4} \%$ per pixel.

The second part of the article deals with the computation of the datum plane, tilt angle and it proposes novel method to roughness analysis. It is based on cosine Fourier series and it clearly computes the distribution of roughness-angles for first-order asperities. This is much more sophisticated than only visual comparison of sample and template graphs. This method can be also applied to roughness-angles for second-order asperities.

Acknowledgement: The authors acknowledge support from Project LO1202 by financial means from the Ministry of Education, Youth and Sports, the National Sustainability Programme I. The author thanks prof. Tomas Ficker from the Faculty of Civil Engineering Brno University of Technology for the provided data. 


\section{References}

[1] Mouralová, K., Kovář, J., Klakurková, L., Bednář, J., Zahradníček, R.: Analysis of the surface morphology and topolography of pure aluminium machined using WEDM, Measurement 114, pp. 169-176, (2018) DOI 10.1016/j.measurement.2017.09.040

[2] Mouralová, K., Matoušek, R., Kovář, J., Mach, J., Klakurová, L., Bednář, J.: Analyzing the surface layer after WEDM depending on the parameters of a machine for the 16MnCr5 steel, Measurement 94, pp. 771-779, (2016) pp. 771-779, DOI 10.1016/j.measurement.2016.09.028

[3] Hoek, E., Brown, E. T.: Underground excavations in rock. London: Institute of Mining and Metallurgy; Spon Press, Taylor \& Francis. (1990)

[4] Hack, R.: Slope Stability Probability Classification (SSPC). ITC publication 43 (2nd ed.). Technical University Delft \& Twente University - International Institute for Aerospace Survey and Earth Sciences (ITC Enschede), Netherlands. pp. 258. (1998).

[5] Price, D. G.: De Freitas, M. H. Engineering Geology: Principles and Practice. Springer. p. 450 (2009).

[6] Hencher, S. R., Lee, S. G., Carter, T. G., Richards, L. R.: Sheeting Joints: Characterization, Shear Strength and Engineering. Rock Mechanics and Rock Engineering. 44 (1), pp. 1-22 (2011). doi:10.1007/s00603-010-0100-y.

[7] Rengers, N.: Influence of surface roughness on the friction properties of rock planes. In: Proceedings 2nd International Congress on Rock Mechanics, ISRM, Belgrade. 1. ISRM. pp. 229-234 (1970).

[8] Fecker, E., Rengers, N.: Measurement of large scale roughness of rock planes by means of profilograph and geological compass. In: Proceedings symposium on rock fracture, Nancy, France. pp. 1-18. (1971).

[9] Hencher, S. R.; Richards, L. R. Laboratory direct shear testing of rock discontinuities. Ground Engineering. 22 (2): pp. 24-31 (1989).

[10] Barton, N. R.; Bandis, S. C. Review of predictive capabilities of JRC-JCS model in engineering practice. Rock Joints: Regional Conference of the International Society for Rock Mechanics ISRM. Loen, Norway: Balkema, Rotterdam, Taylor \& Francis. pp. 603-610 (1990).

[11] Phien-wej, N., Shrestha, U. B.: Rantucci, G. Effect of infill thickness on shear behavior of rock joints. Rock Joints. Balkema, Taylor \& Francis, Rotterdam. pp. 289-294. (1990).

[12] Hack, R., Price, D., Rengers, N.: A new approach to rock slope stability - a probability classification Bulletin of Engineering Geology and the Environment. 62 (2): pp. 167-184 (2003) DOI 10.1007/s10064-002-0155-4.

[13] Ulusay, R., Hudson, J. A.: The Blue Book - The Complete ISRM Suggested Methods for Rock Characterization, Testing and Monitoring: 1974-2006. Ankara: ISRM \& ISRM Turkish National Group. p. 628. (2007)

[14]Laubscher, D. H.: A geomechanics classification system for rating of rock mass in mine design. Journal South African Institute of Mining and MetaEqllurgy. 90 (10): pp. 257-273 (1990). DOI 10.1016/0148-9062(91)90830-F

[15] Neuendorf, K. K. E., Mehl, J. P., Jackson, J. A.: Glossary of Geology, Fifth edition, American Geological Institute (2005)

[16] Hoek, E.; Bray, J. Rock slope engineering. London: Institute of Mining and Metallurgy. pp. 358. (1974).

[17]Xian-Qin, H.; Cruden, D. M. A portable tilting table for on-site tests of the friction angles of discontinuities in rock masses. Bulletin of the International Association of Engineering Geology. 46(1), pp. $59-62$ (1992) DOI: 10.1007/BF02595034

[18] Duncan C. W., Mah, C.: Rock Slope Engineering. Fourth Edition, CRC Press (2004)

[19] Avsar, E. O et al.: Determining Roughness Angle of Limestone Using Optical Laser Scanner, International Journal of Environment and Geoinformatics Vol 3(3), pp. 57-75 (2016).

[20] Martišek, D., Druckmüllerová,H.: Registration of partially focused images for $2 D$ and $3 D$ reconstruction of oversized samples, Scanning (2017) DOI 10.1155/2017/8538215

[21] Wyawahare, M., V., et al.: Image Registration Techniques: An overview, International Journal of Signal Processing, Image Processing and Pattern Recognition, 2 (3), pp. 11-27 (2009).

[22] Martišek, D., et al.: High-quality three-dimensional reconstruction and noise reduction of multifocal images from oversized samples, Journal of Electronic Imaging, 24 (5), (2015). DOI 10.1117/1.JEI.24.5.053029

[23] Martišek, D.: The 2-D and 3-D processing of images provided by conventional microscopes, Scanning, 24(6), pp. 284-296, (2002)

[24] Martišek, D.: 3D Reconstruction of the Surface Using a Standard Camera, Mathematical problems in Engineering, Article Number: 4642397. (2017) DOI 10.1155/2017/4642397

[25] Martišek, D., Procházková, J.: The Enhancement of 3D Scans Depth Resolution Obtained by Confocal Scanning of Porous Materials, Measurement Science Review, 17 (6), pp. 273-281 (2017) DOI 10.1515/msr-2017-0034

[26] Matousek, R., Popela, P. Kudela, J.: Heuristic Approaches to Stochastic Quadratic Assignment Problem: VAR and CVAR Cases, MENDEL, vol. 23, No. 1, pp. 73-78, Brno (June 2017), ISSN 1803-3814

[27] Starha, P., Matousek, R., Zikmund, T.: Measuring Velocity of Passing Vehicle by utilizing Image Processing, MENDEL, vol. 21, pp. 165-168, Brno (June 2015), ISSN 1803-3814

[28] Starha, P., Martisek, D., Matousek, R.: Numerical Methods of Object Reconstruction using the Method of Moments, MENDEL, vol. 20, pp. 241-248, Brno (June 2014), ISSN 1803-3814 